\title{
Conjoint Statement of the SNM, ACNM, and ABNM on Credentialing and Delineation of Privileges for Therapeutic Procedures Using Radiopharmaceuticals
}

\section{EXECUTIVE SUMMARY}

The training requirements for the supervision and administration of therapeutic procedures using radiopharmaceuticals are not defined uniformly in the Accreditation Council of Graduate Medical Education (ACGME) program requirements for different specialties. The training requirements of the Nuclear Regulatory Commission (NRC) are intended for the safe handling of radioisotopes, which is only a prerequisite to the safe practice of medicine.

The purpose of this document is to recommend the minimum training requirements for therapeutic procedures using radiopharmaceuticals. These requirements should be uniform across ACGME specialty training requirements. This training must include independently evaluating and treating, under supervision, a minimum of 10 cases of radioiodine therapy for hyperthyroidism, a minimum of 5 cases of radioiodine therapy for thyroid carcinoma, and a minimum of 3 cases of parenteral radionuclide therapy. Less training and experience is inadequate to ensure the safety, appropriateness, and effectiveness of radionuclide therapy.

\section{GENERAL}

A. The Joint Commission (formerly known as the Joint Commission for the Accreditation of Hospitals) requires that a system be in place for delineating privileges for every hospital staff member. The Joint Commission does not, however, spell out specific qualifications for any given privilege or level of privilege. Privileges are generally hospital-specific and are not usually transferable from hospital to hospital.

B. The granting of clinical privileges cannot and should not depend on only a single criterion, such as board certification or membership in a particular specialty society. Other criteria should also be used, such as documented evidence of training, experience, and judgment and demonstrated current competence.

C. It is the final responsibility of the hospital medical staff and hospital governing board to ensure that a physician meets a reasonable standard of competency.

D. The SNM, American College of Nuclear Medicine (ACNM), and American Board of Nuclear Medicine (ABNM) have reviewed the current ACGME training requirements for nuclear medicine, radiology, nuclear radiology, and radiation oncology and the guidelines

COPYRIGHT $\odot 2011$ by the Society of Nuclear Medicine, Inc. DOI: $10.2967 /$ jnumed.110.084962 from other specialty societies and endorse the recommendations given in section II.D below.

\section{SUMMARY OF CURRENT REQUIREMENTS FOR THERAPEUTIC PROCEDURES USING RADIOPHARMACEUTICALS}

A. Certification requirements for physicians supervising and administering therapeutic procedures using radiopharmaceuticals: Physicians supervising and administering therapeutic procedures using radiopharmaceuticals must be certified by one of the following specialty boards of the American Board of Medical Specialties, which include training for therapeutic procedures using radiopharmaceuticals:

1. The ABNM

2. The American Board of Radiology (certification in diagnostic radiology with a subspecialty in nuclear radiology, or certification in radiation oncology, or certification in diagnostic radiology)

B. NRC requirements for authorized user status for use of therapeutic radionuclides: The NRC training and experience requirements for becoming an authorized user of unsealed byproduct material are described in U.S.NRC 35.390 (http://www.nrc.gov/reading-rm/doccollections/cfr/part035/part035-0390.html). These requirements include "Administering dosages of radioactive drugs to patients or human research subjects involving a minimum of three cases in each of the following categories for which the individual is requesting authorized user status-

“(1) [35.392] Oral administration of less than or equal to 1.22 gigabecquerels ( 33 millicuries) of sodium iodide I-131, for which a written directive is required;

“(2) [35.394] Oral administration of greater than 1.22 gigabecquerels (33 millicuries) of sodium iodide I$131 \ldots$;

“(3) [35.396] Parenteral administration of any beta emitter, or a photon-emitting radionuclide with a photon energy less than $150 \mathrm{keV}$, for which a written directive is required; and/or

“(4) [35.396] Parenteral administration of any other radionuclide, for which a written directive is required..."

C. ACGME program requirements for therapeutic procedures using radiopharmaceuticals: ACGME has the 
following program requirements for therapeutic procedures using radiopharmaceuticals.

1. Nuclear medicine (http://www.acgme.org/acWebsite/ RRC_200/200_prIndex.asp) (July 2007)

a. Section Int.A. "Nuclear Medicine is the clinical and laboratory medical specialty that uses radioactive and stable tracers to study physiologic, biochemical and cellular processes for diagnosis, therapy and research."

b. Section Int.C. “. . . Residents should be qualified to recommend therapy....If radionuclide therapy is indicated, they should be capable of assuming responsibility for patient care..."

c. Section II.D.5. "Teaching case files involving diagnostic and therapeutic nuclear medicine procedures should be available and should cover the full spectrum of clinical applications.... There must be a mechanism for maintaining case records and treatment results to facilitate patient follow-up...."

d. Section IV.A.5.a).(1) "Residents must be able to provide patient care....Residents...will obtain patient information relevant to the requested test or therapy using patient interview, chart and computer data base review, physical examination, and contact with referring physician;"

e. Section IV.A.5.a).(2) Residents "will select appropriate procedures or therapy based on the referring physician's request and the patient's history."

f. Section IV.A.5.a).(4) Residents "will conduct therapeutic procedures. Therapeutic procedures must be done in consultation with an attending physician who is a licensed user of radioactive material. These procedures should include dose calculation, patient identity verification, explanation of informed consent, documentation of pregnancy status, counseling of patients and their families on radiation safety issues, and scheduling follow-up after therapy."

g. Section IV.A.5.a).(5) Residents "will maintain records (logs) of participation. . in all types of therapy procedures;"

h. Section IV.A.5.a).(6) Residents "should attain sequentially increasing competence in...treating and following up the patient who receives radionuclide therapy. Under adequate faculty supervision, the resident should participate directly in the performance of. . .therapeutic procedures;"

i. Section IV.A.5.a).(8).(d) “. . .Thyroid studies should include measurement of iodine uptake and dosimetry calculations for radio-iodine therapy;"

j. Section IV.A.5.a).(8).(k) Residents must have experience with "therapeutic administration of radiopharmaceuticals, to include patient selection and understanding and calculation of the administered dose. Specific applications should include radioiodine in hyperthyroidism (minimum of 10 cases) and thyroid carcinoma (minimum of five cases), radiolabeled antibodies (minimum of three cases)..." Parenteral radionuclide therapy currently includes radiolabeled antibodies for the treatment of lymphoma, radionuclide for the treatment of bone metastasis and radiolabeled microspheres for the treatment of liver metastasis. "Program directors must be able to document the experience of residents in this area, including patient follow-up, (e.g., with logbooks);"

k. Section IV.A.5.b).(1) Residents "will closely follow scientific progress in nuclear medicine, and learn to incorporate it effectively for modifying and improving diagnostic and therapeutic procedures;"

1. Sections IV.A.5.b).(7), IV.A.5.b).(9), and IV.A.5. b).(9).(d) Residents "must have didactic instruction in...therapeutic applications..." including "therapeutic uses of unsealed radiopharmaceuticals: patient selection and management, including dose administration and dosimetry, radiation toxicity, and radiation protection considerations in the treatment of metastatic cancer and bone pain, primary neoplasms, solid tumors, and malignant effusions; and the treatment of hematologic, endocrine, and metabolic disorders..."

The ABNM requires documentation by the program director that the candidate has successfully completed this training. The residency program must include the training and experience required by the NRC as described in 10 Code of Federal Regulations 35.190, 35.290, and 35.390. The candidate must have been judged competent to practice clinical nuclear medicine and must pass a comprehensive, secure, psychometrically valid, computer-based certification examination. The ABNM Web site (http://www.abnm.org) states that the diplomate is capable of serving as a consultant to other physicians, advising them of the clinical indications and limitations as well as benefits versus risks of diagnostic and therapeutic applications of molecular imaging and molecular medicine.

2. Nuclear radiology (http://www.acgme.org/acWeb site/RRC_420/420_prIndex.asp) (January 2005)

a. Section 1.A. The scope of the specialty is defined as "a clinical subspecialty of radiology involving diagnostic and therapeutic use of radioactive materials using unsealed sources."

b. Section V.A.1. "The training program shall include graduated study, experience, and responsibility in all facets of nuclear radiologic diagnosis and therapy." 
c. Section V.B.1. "Formal instruction is required in...therapeutic administration of radionuclides...."

The number and types of treatment required are not stated.

3. Radiation oncology (http://www.acgme.org/acWebsite/ downloads/RRC_progReq/430_radiation_oncology_ 01012009.pdf) (January 2009)

a. Section IV.A.5.a).(3) The residents "must participate in the administration of no fewer than six procedures using radioimmunotherapy, other targeted therapeutic radiopharmaceuticals, or unsealed radioactive sources;"

b. Section IV.A.5.a).(7) " . . the training program must provide instruction in the physics, radiation and cancer biology, and clinical applicability of the following areas:...radioimmunotherapy, unsealed sources...."

c. Section IV.A.5.b).(2) The training program "must have a curriculum in medical physics that includes instruction and practical demonstrations of. .the safe handling of sealed and unsealed radionuclides."

4. Diagnostic radiology (http://www.acgme.org/ acWebsite/downloads/RRC_progReq/420_diagnostic_ radiology_07012008.pdf) (July 2008)

a. Section Int.A. The scope of the specialty encompasses "image guided therapeutic techniques. ..." Sections IV.A.5.a).(2) and IV.A.5.a).(2).(a) Residents "must have a minimum of 700 hours (approximately 4 months) of training and experience in clinical nuclear medicine. ...Each resident must participate with preceptors in at least three therapies involving oral administration of I131. Documentation of the resident's participation must include the date, diagnosis, and dose of each I-131 therapy;",

b. Sections IV.A.5.b).(1).(a).(iii) and IV.A.5.b).(1).(a). (iv). "There must be at least 80 hours of didactic (classroom and laboratory training) training under the direction of an authorized user (AU). This training must include the following subjects as they relate to nuclear medicine:...safe handling and administration of therapeutic doses of unsealed radionuclide sources (i.e., I-131)..."

c. The American Board of Radiology requires candidates who seek certification with authorized user eligibility to have participated in at least 3 low-dose $(\leq 33 \mathrm{mCi})$ and 3 high-dose $(>33 \mathrm{mCi}){ }^{131} \mathrm{I}$ therapies. The American Board of Radiology requires documentation by the program director and qualified authorized users that the candidate has successfully completed this training. The residency program must include training and experience regarding the requirements of the Nuclear Regulatory Commission as described in parts 10 CFR 35.290, 10 CFR 35.392, and 10 CFR 35.394. The diagnostic radiology program requirements have not been changed to reflect this new American Board of Radiology requirement. There is currently no mechanism for the American Board of Radiology to recognize an authorized user eligible for 290 or 392 uses only.

D. The SNM, ACNM, and ABNM believe that the minimum training requirements of the $\mathrm{NRC}$ for the handling and use of radiopharmaceuticals are intended for the radiation safety of patients, health care workers, and the public. The medical treatment of patients with therapeutic radiopharmaceuticals for benign and malignant diseases involves considerably more than just radiation safety. It requires extensive training and experience that should include the following:

1. Review of the request form and patient medical record

2. Review of relevant laboratory values, including pregnancy testing, if appropriate

3. Review of imaging studies as appropriate

4. Interview of the patient to obtain history

5. Examination of the patient as appropriate

6. Full explanation of the procedure, answering all patient questions (including risks and radiation safety precautions) and obtaining informed consent

7. Calculation of the dose to be administered

8. Supervision of administration of the dose to the patient

9. Arrangement for appropriate follow-up

The SNM, ACNM, and ABNM believe that the ACGME nuclear medicine program requirements are minimum requirements for safe supervision and effective administration of therapeutic procedures using radiopharmaceuticals, especially for patients who are being treated for cancer. Training and experience that do not fulfill ACGME nuclear medicine program requirements are inadequate to ensure the safety and effectiveness of radionuclide therapy. The minimum requirements for supervision and administration of therapeutic procedures using radiopharmaceuticals (defined in the ACGME Nuclear Medicine Program Requirements) include independently evaluating and treating, under supervision, a minimum of 10 cases of radioiodine therapy for hyperthyroidism, a minimum of 5 cases of radioiodine therapy for thyroid carcinoma, and a minimum of 3 cases of parenteral radionuclide therapy. Additional experience can be gained after residency training under the supervision of a qualified physician if 


\begin{tabular}{|c|c|c|}
\hline Type of therapy & Board certification & $\begin{array}{l}\text { Total clinical } \\
\text { experience required* }\end{array}$ \\
\hline \multirow{4}{*}{$\begin{array}{l}\text { Oral administration of sodium }{ }^{131} \mathrm{I} \leq 1.22 \mathrm{GBq} \\
(33 \mathrm{mCi})[35.392]\end{array}$} & 1. ABNM & 10 cases \\
\hline & $\begin{array}{l}\text { 2. ABR with certificate of added qualifications } \\
\text { in nuclear radiology }\end{array}$ & 10 cases \\
\hline & 3. ABR with certification in radiation oncology & 10 cases \\
\hline & $\begin{array}{l}\text { 4. ABR with certification in diagnostic radiology } \\
\text { with "authorized user-eligible" designation }{ }^{\dagger}\end{array}$ & 10 cases \\
\hline \multirow{3}{*}{$\begin{array}{l}\text { Oral administration of sodium }{ }^{131} \mathrm{I}>1.22 \mathrm{GBq} \\
\quad(33 \mathrm{mCi})[35.394]\end{array}$} & 1. ABNM & 5 cases \\
\hline & $\begin{array}{l}\text { 2. ABR with certificate of added qualifications } \\
\text { in nuclear radiology }\end{array}$ & 5 cases \\
\hline & 3. ABR with certification in radiation oncology & 5 cases \\
\hline \multirow{3}{*}{$\begin{array}{l}\text { Parenteral administration of any } \beta \text {-emitter, } \\
\text { photon emitter }<150 \mathrm{keV} \text {, or other } \\
\text { radionuclide requiring a written directive } \\
\text { [35.396] }\end{array}$} & 1. ABNM & 3 cases \\
\hline & $\begin{array}{l}\text { 2. ABR with certificate of added qualifications } \\
\text { in nuclear radiology }\end{array}$ & 3 cases \\
\hline & 3. ABR with certification in radiation oncology & 3 cases \\
\hline
\end{tabular}

*Includes experience for authorized user. Section II.C.1.j of the text explains minimum requirements for independently evaluating and treating patients under supervision. Clinical experience may be gained after residency training under the supervision of a qualified physician.

${ }^{\dagger}$ This ABR certificate is recognized as fulfilling the NRC requirements for safe handling and oral administration of ${ }^{131} 1 \leq$ and $>33 \mathrm{mCi}$ (1.22 GBq).

10 Code of Federal Regulations part and section are presented in brackets.

$\mathrm{ABR}=$ American Board of Radiology.

experience during residency training does not meet these recommendations. Such supplementation will count toward fulfilling the minimum numbers of case experiences recommended in this statement.

E. Physicians need to participate in maintenance of certification as required by the specialty board. There needs to be evidence of continuing competence in the safe supervision and effective administration of therapeutic procedures using radiopharmaceuticals.

\section{GENERAL PROCEDURES FOR CREDENTIALING PROCESS}

A. It is recommended that any physician applying for privileges to practice nuclear medicine, including supervision and administration of therapeutic procedures using radiopharmaceuticals in either a hospital or clinical setting, should document proper credentials, including evidence of training and continuing maintenance of certification, as defined above.

B. The following criteria are recommended for delineating privileges for radiopharmaceutical therapy (Table 1):

1. Graduation from a Liaison Committee on Medical Education-approved medical school or school of osteopathy, or graduation from a foreign medical school with possession of an Educational Commission for Foreign Medical Graduates (ECFMG) certificate score acceptable for medical licensure in the state of medical practice

2. Satisfactory completion of an approved training program in nuclear medicine, nuclear radiology, radiation oncology, diagnostic radiology, or the equivalent, plus clinical experience as recommended in II.D and Table 1

3. Clinical competence, appropriate for medical practice; at present, current competency may be demonstrated by one of the following:

a. Documentation of completion of an examination and issuance of a certificate from a recognized certifying organization such as an appropriate American Board of Medical Specialties-recognized specialty board, or evidence of recertification within the past $10 \mathrm{y}$

b. Maintenance of certification as required by the appropriate American Board of Medical Specialties-recognized specialty board

4. A method of review for regular delineation of privileges as required by individual institutions annually

5. Definition of which individual procedure or category of procedures may be performed by each physician

\section{APPROVAL}

This statement was approved by the board of directors of the SNM on September 12, 2010; the board of directors of the ACNM on September 29, 2010; and the board of directors of the ABNM on October 26, 2010.

\section{WRITING GROUP}

Dominique Delbeke, Michael Graham, Henry Royal, George Segall, Barry L. Shulkin, and Harvey A, Ziessman. 